\title{
Expression of PRDX6 Correlates with Migration and Invasiveness of Colorectal Cancer Cells
}

\author{
Wen-Shih Huang ${ }^{a, b} \quad$ Cheng-Yi Huang ${ }^{a}$ Meng-Chiao Hsieha,c Yi-Hung Kuo ${ }^{a, c}$ \\ Shui-Yi Tung,d Chien-Heng Shenc,d Yung-Yu Hsieh ${ }^{d}$ Chih-Chuan Teng ${ }^{\text {e,f }}$ \\ Ko-Chao Lee ${ }^{g}$ Kam-Fai Lee ${ }^{h}$ Hsing-Chun Kuo ${ }^{\text {ef,i,j }}$
}

aDivision of Colon and Rectal Surgery, Department of Surgery, Chang Gung Memorial Hospital, Chiayi, ${ }^{b}$ Chang Gung University College of Medicine, Taoyuan, 'Graduate Institute of Clinical Medical Sciences, College of Medicine, Chang Gung University, Taoyuan, dDepartment of Hepato-Gastroenterology, Chang Gung Memorial Hospital, Chiayi, elnstitute of Nursing and Department of Nursing, Chang Gung Institute of Technology Chiayi Campus, Chiayi, 'Research Fellow, Chang Gung Memorial Hospital, Chiayi, ${ }^{9}$ Department of Colorectal Surgery, Department of Surgery, Chang Gung Memorial Hospital

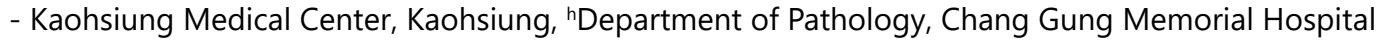
at Chiayi, Chiayi, 'Chronic Diseases and Health Promotion Research Center, CGUST, Chiayi, ${ }^{\mathrm{j} R e s e a r c h}$ Center for Industry of Human Ecology and Research Center for Chinese Herbal Medicine, College of Human Ecology, Chang Gung University of Science and Technology, Taoyuan, Taiwan

\section{Key Words}

Colorectal cancer (CRC) • PRDX6 • Tumorigenicity • PI3K/AKT/p38/p50

\begin{abstract}
Background/Aims: Colorectal cancer (CRC) is the third most common type of cancer and the second leading cause of cancer-related deaths worldwide. PRDXs are antioxidant enzymes that play an important role in cell differentiation, proliferation and apoptosis and have diverse functions in malignancy development. However, the mechanism of aberrant overexpression of PRDX6 in CRC remains unclear. Methods: Boyden chamber assay, flow cytometry and a lentiviral shRNA targeting PRDX6 and transient transfection with PCMV-6-PRDX6 plasmid were used to examine the role of PRDX6 in the proliferation capacity and invasiveness of CRC cells. Immunohistochemistry (IHC) with tissue array containing 40 paraffin- embedded CRC tissue specimens and Western blot assays were used to detect target proteins. Results: PRDX6 was significantly up-expressed in different comparisons of metastasis of colorectal adenomas in node-positive CRC $(P=0.03)$. In in vitro HCT-116, PRDX6 silencing markedly suppressed CRC cell migration and invasiveness while also inducing cell cycle arrest as well as the generation of reactive oxygen species (ROS); specific overexpression of PRDX6 had the opposite effect. Mechanistically, the PRDX6 inactivation displayed decreased levels of PRDX6,
\end{abstract}

W.-S. Huang and C.-Y. Huang contributed equally to this work.

Kam-Fai Lee Department of Pathology, Chang Gung Memorial Hospital;

and Hsing-Chun Kuo, Ph.D. Chang Gung University of Science and Technology, Chia-Yi Campus, Chiayi (Taiwan)

Tel.+886-5-3628800, Fax 886-5-3628866, E-Mail guscsi@gmail.com 
Huang et al.: PRDX6 Expression May Participate in the Regulation of the CRC

Tumorigenicity

$\mathrm{N}$-cadherin, $\beta$-catenin, Vimentin, Slug, Snail and Twist-1 through the activation of the PI3K/ AKT/p38/p50 pathways, but they were also significantly inhibited by PRDX6 transfectants. There was also increased transcriptional activation of dimethylation of histone $\mathrm{H} 3$ lysine 4 (H3K4me3) of PRDX6 promoter via the activation of the PI3K/Akt/NFkB pathways. Conclusion: Our findings demonstrated that PRDX6 expression plays a characteristic growth-promoting role in CRC metastasis. This study suggests that PRDX6 may serve as a biomarker of nodepositive status and may have a role as an important endogenous regulator of cancer cell tumorigenicity in CRC. PRDX6 may also be an effective therapeutic target.

(C) 2018 The Author(s)

Published by S. Karger AG, Basel

\section{Introduction}

Colorectal cancer, one of the most common cancers worldwide, is frequently categorized as a leading cause of cancer-related deaths. Death due to colorectal cancer, which arises from epithelial cells, usually results from uncontrolled metastatic disease [1]. Only a minority of CRC cases are detected at a stage early enough for potential treatment with medicinal therapies such as surgery, chemotherapy, and radiotherapy. Rapid tumor growth has the features of rapid progression and poor outcome [2], which is the major problem affecting CRC therapy; however, patients with vascular and lymph node metastases are not eligible for surgical therapy. The presence of positive lymph nodes, which distinguishes stage I/II from stage III CRC, is a particularly key factor in patient management [3]. During this period, the progression of CRC appears to follow a precise series of molecular events, requiring the accumulation in proto-oncogenes and aberrant tumor suppressor genes in these initially benign lesions [4]. Access to specimens of CRC at different stages of the malignancy has allowed the analysis of the molecular alterations most frequently associated with each step of the CRC [5]. Several clinical and experimental studies have shown that a loss of E-cadherin at the cell membrane surface is a key hallmark of epithelial-mesenchymal transition (EMT), and these studies have described a reverse relationship between E-cadherin and tumorigenicity [6]. In addition, EMT occurs widely in tumor cell invasion and metastasis, and the Phosphatidylinositol-3-kinase (PI3K)/Akt pathway has been shown to be critical in promoting cell survival, inhibiting apoptosis, and creating tumorigenicity (You et al., 2009; Shin et al., 2010; Chen et al., 2012) [7]. These observations have led to studies on the potential mechanism for the association between EMT induction and the development of CRC metastasis, and to discussions concerning new biomarkers of node-positive status that underlie the signaling pathways that are altered in CRC and that are responsible for the initiation and progression [8].

Peroxiredoxins (PRDXs), a ubiquitously expressed family of small (22-27 $\kappa \mathrm{Da})$ nonseleno peroxidases that catalyze the peroxide reduction of H2O2, organic hydroperoxides and peroxynitrite, have emerged as having important physiological functions, including growth, survival, migration, and differentiation [9]. Several studies have indicated that PRDXs overexpression in cancer tissues strongly correlates with a more aggressive cancer behavior, tumor metastasis and the tumor-node-metastasis (TNM) stage, indicating a possible role in tumorigenesis progression [10]. PRDXs contain protein composed of 6 mammalian PRDXs, namely PRDX I to VI [11]. PRDX6 is expressed in almost all mammalian tissues and is highly expressed in solid organs. In some cancers, which might be attractive for solid organ tumorigenesis, PRDX6 created an altered redox environment between oxidant balance and cancer [12]. These data suggest that the mechanism by which PRDX6 exerts its oncogenic action in CRC is still poorly understood, and further studies are required.

Previous studies have indicated that CRC metastasis is a multistage process [12]. A previous report demonstrated that EMT is a transcriptional program that occurs normally during embryonic development and is characterized by changes in the expression levels for E-cadherin, a mediator of cell-cell adhesion, and other markers characteristic of mesenchymal and epithelial cells. In addition, another process that is frequently associated with metastasis is the epithelial-to-mesenchymal transition (EMT) [13]. A downstream effector of MAPK in 
Huang et al.: PRDX6 Expression May Participate in the Regulation of the CRC

Tumorigenicity

response to EMT is phosphorylated on PI3K/AKT/p38 pathways and regulates cell cycle progression by controlling the transcriptional output of several key genes, including CDK2 with Cyclin E and CDK4 with Cyclin D1 [14]. However, no studies have documented a role for the PRDX6 signaling axis in the regulation of CRC cell growth. In the present study, we investigated whether experimental manipulation of PRDX6 expression in established CRC cell lines influenced invasion, survival, and EMT. We showed that the expression of PRDX6 was upregulated in response to the activation of the PI3K/AKT/p38 pathways' intracellular signaling cascades through histone H3 lysine 4 (H3K4me3) of the PRDX6 promoter, a wellknown marker of transcriptional activation [15]. Our findings provide evidence of the molecular mechanism by which PRDX6 mediates an invasiveness pathway in colorectal cancer cells, suggesting associations of PRDX6 with lymph node metastasis.

\section{Materials and Methods}

\section{Materials}

All culture materials were purchased from Gibco (Grand Island, NY, USA). 3-(4, 5-dimethylthiazol2-yl)-2, 5-diphenyltetrazolium bromide (MTT), ROS scavenger (N-acetyl cysteine [NAC]), 2, 7-dichlorodihydrofluorescein diacetate (H2DCFDA), dihydroethidium (DHE), phosphoinositide 3-kinase inhibitor (wortmannin) and p38 MAPK inhibitor (SB203580) were purchased from Sigma (St. Louis, MO, USA). Mouse monoclonal antibodies against PRDX6, N-cadherin, b-catenin, Vimentin, Slug, Snail, Twist-1 and acetylation of H3 (Ac-Histone H3) at Lys 9 and Lys 14, and $\beta$-actin were purchased from Santa Cruz Biotechnology (Santa Cruz, CA, USA). Rabbit polyclonal antibodies against ERK1/2Thr ${ }^{202} \mathrm{Ty}^{\mathrm{r204}}$, p38 $\mathrm{Thr}^{180} \mathrm{Tyr}^{182}$, and phosphoinositide 3-kinase $\mathrm{Tyr}^{458}$ mouse/rabbit monoclonal antibody were purchased from Cell Signaling Technology (Beverly, MA, USA). The TdT-mediated dUTP Nick End Labeling (TUNEL) kits were from Roche (Germany). SDS, NP-40, sodium deoxycholate, protease inhibitor cocktails were purchased from Sigma (St. Louis, MO, USA). SDS, NP-40, sodium deoxycholate, protease inhibitor cocktails were purchased from Sigma.

\section{Tissue samples and assessment of CRC tissue microarrays (TMAs)}

Tissues from 40 cases of primary colorectal cancer from the Chang Gung Memorial Hospital, Chiayi, Taiwan, were surgically resected. Forty patients with CRC (18 node-negative, 22 node-positive), who underwent surgery without presurgical chemotherapy or radiation therapy from 2003 to 2006, were included (Table 1). The institutional review boards of the Chang Gung Memorial Hospital approved the study (IRB103-2992C/CGMH), and informed consent was obtained from all patients and the tissue bank, department of medical research. Written informed consent was obtained from each patient before surgery. The Ethics Committee of Chang Gung Memorial Hospital and our institute approved the protocol. The excised samples were obtained from polyp and cancer tissues within one hour of surgery. All excised tissues were immediately placed in liquid nitrogen and stored at $-80^{\circ} \mathrm{C}$ for further analyses. The pathologist confirmed diagnosis of all samples used in the study. TMA used in the confirmatory studies represented 94 cases of eligible CRC specimens (14 node-negative, 22 node-positive) and was used for immunohistochemistry with anti-PRDX6 antibody. This TMA contained carcinoma samples in duplicate, with normal control colonic mucosa [16]. Immunostaining was assessed independently by a pathologist in a blinded manner. The staining of PRDX6 was scored as the product of the staining intensity (on a scale of $0-2$ : negative $=0$, low $=1$, high $=2$ ) and the percentage of cells stained (on a scale of 0-3: $0=$ zero, $1=1 \%-25 \%, 2=26 \%-$ $50 \%, 3=51 \%-100 \%)$, resulting in scores on a scale of $0-5$ [17].

Table 1. Baseline characteristics of patients with colorectal cancer

\begin{tabular}{lc}
\hline Parameter & Cases \\
\hline Gender & 21 \\
Male & 19 \\
Female & $64.8(27-89)$ \\
Baseline characteristics & \\
Tumor location & 28 \\
Colon & 12 \\
Rectum & 4.2 \\
Average tumor diameter (cm) & \\
Differentiation degree & 29 \\
Moderately or poorly differentiated & 11 \\
Highly differentiated & \\
TNM staging & 18 \\
I + II stage & 22 \\
III + IV stage & \\
\hline
\end{tabular}




\section{Cellular Physiology Cell Physiol Biochem 2018;51:2616-2630 \\ and Biochemistry \begin{tabular}{l|l} 
Dublished onlIne: T1 December 2018 & $\begin{array}{l}\text { (c) } 2018 \text { The Author(s). Published by S. Karger AG, Basel } \\
\text { www.karger.com/cpb }\end{array}$ \\
\hline
\end{tabular} \\ Tumorigenicity}

Cell culture

Human colon cancer cell line DLD-1 (CCL-221) and human colorectal carcinoma cell line HCT-116 (CCL-247) were purchased from American Type Culture Collection (ATCC). DLD-1 cells were cultured in RPMI 1640 medium composed of 10\% fetal calf serum (FCS) (S0113; Biochrom KG, Berlin, Germany) and $1 \%$ antibiotics (100 units/mL of penicillin and $100 \mu \mathrm{g} / \mathrm{mL}$ of streptomycin) (Sigma Chemicals, St. Louis, MO, USA) and incubated at $37^{\circ} \mathrm{C}$ with $5 \% \mathrm{CO}$. Adhered cells were washed twice with PBS. HCT-116 was cultured in DMEM supplemented with $10 \%$ heat-inactivated newborn calf serum at $37^{\circ} \mathrm{C}$ in a humidified $5 \%$ CO2 incubator [18].

\section{Generation of stably and transiently expressing PRDX6 colorectal cancer cell clone}

HCT-116 cells were transfected with PRDX6 short hairpin RNA (shRNA) (sc-40835-v) or scrambled shRNA (sc-108084; purchased from Santa Cruz Biotechnology) lentiviral particles in $0.5 \mathrm{~mL}$ of serum-free media, then selected in $10 \mathrm{lg} / \mathrm{mL}$ puromycin for an additional 10 days (sc-108071; Santa Cruz Biotechnology). Surviving cell colonies were picked and cultured before testing for PRDX6 expression. In addition, for transient transfection, the cells $\left(5 \times 10^{5}\right)$ were plated in 6 -well plates and transfection was performed with pCMV-6-PRDX6 tag plasmids, purchased from Origene, in serum-free medium that contained Lipofectamine Plus (Invitrogen, Carlsbad, CA, USA). Transfection efficiency was assessed by Western blot analysis [19, 20].

\section{Cell cycle distribution analysis and reactive oxygen species}

Changes in cell morphological characteristics during apoptosis were examined using fluorescence microscopy of 4',6-diamidino-2-phenylindole (DAPI)-stained cells. The monolayer of cells was fixed with 4\% paraformaldehyde for $30 \mathrm{~min}$ at room temperature. The fixed cells were permeabilized with 3 treatments in $0.2 \%$ Triton X-100 in phosphate-buffered saline, followed by incubation with $1 \mu \mathrm{g} / \mathrm{mL}$ of DAPI for 30 min. The apoptotic nuclei were detected under 200× magnification using a fluorescent microscope with a 340/380 nm excitation filter and were scored according to the percentage of apoptotic nuclei found in samples containing 200 to 300 cells. Cell-cycle distribution was analyzed using flow cytometry. Cells stained with propidium iodide were analyzed with a FACScalibur ${ }^{\mathrm{TM}}$ (Becton Dickinson), and the data were analyzed using a mod-fit cell cycle analysis program [21].

The intracellular accumulation of ROS was determined by using the fluorescent probes of $10 \mu \mathrm{M} \mathrm{2}$ ', 7'-dichlorofluorescein diacetate (H2DCFDA, Molecular Probes) for the measurement of hydrogen peroxide (H202), and the cells were washed prior to FACS analysis. Cell Quest software (Becton Dickenson) was described earlier. The results were presented as a percentage of the fluorescent intensity compared with the control sample [22].

\section{Preparation of total cell extracts and immunoblot analyses}

Cells were lysed with a buffer containing 1\% NP-40, $0.5 \%$ sodium deoxycholate, $0.1 \%$ sodium dodecyl sulfate (SDS), and a protease inhibitor mixture (phenylmethylsulfonyl fluoride, aprotinin, and sodium orthovanadate). The total cell lysate (50 $\mathrm{\mu g}$ of protein) was separated by SDS-polyacrylamide gel electrophoresis (PAGE) (12\% running, 4\% stacking) and analyzed by using the designated antibodies and the Western-Light chemiluminescent detection system (Bio-Rad, Hercules, CA, USA). The area of the photo images in immunoblot was determined by measuring the numbers of pixels using ImageGauge 3.46 software (Fujifilm, Inc.) as previously described [23]. Through quantitative analysis of fluorescence assays using ImageJ, images were captured on the hard drive of the workstation computer [22].

\section{Matrigel invasion assay and scratch assays}

A straight wound line, a scratch $(4 \mathrm{~mm})$, was made in the monolayer of the cells by plating cells in a 6-well culture dish. The cells were transfected with lentiviral shRNA targeting PRDX6 (siPRDX6) or PRDX6 plasmid, and the wound line was monitored for $24 \mathrm{~h}$. Pictures of cells along the wound line were taken by phase-contrast microscope, and Openlab v3.0.2 image analysis software (Improvision, Coventry, UK) was used to quantify the area progressively filled with cells over the period of the experiment [23].

The Boyden chamber assay used for the analysis of tumor cell invasion is based on a chamber with two medium-filled compartments as described before..$^{19}$ Cells $\left(1 \times 10^{5} / \mathrm{ml}\right)$ in serum-free medium were added to an inner cup of the 48-well Transwell chamber (Corning Life Sciences, Corning, NY, USA) that had been coated with $50 \mu \mathrm{l}$ of Matrigel (BD Biosciences, Franklin Lakes, NJ, USA; in serum-free medium). Medium 


\section{Cellular Physiology Cell Physiol Biochem 2018;51:2616-2630

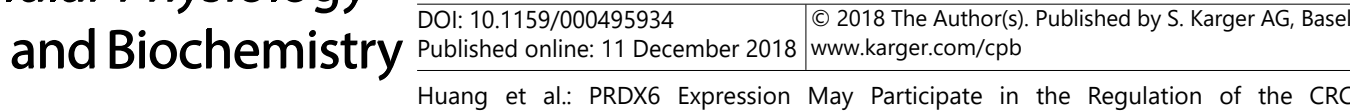 \\ Tumorigenicity}

supplemented with $10 \%$ serum or indicated agent was added to the outer cup. Cells were allowed to migrate for $24 \mathrm{~h}$, and then the membrane was fixed and stained with modified Giemsa stain (Sigma-Aldrich). The human HCT-116 cells and DLD-1 cells on the lower side of the membrane were counted using a light microscope at 200x magnification. The number of cells that migrated to the lower side of the membrane was determined. ${ }^{13}$ Five random fields that migrated to the lower side of the membrane in each well were counted, and the average number/field from triplicated wells was plotted [24].

\section{Chromatin immunoprecipitation (ChIP) analysis}

HCT-116 cells were cross-linked with $1 \%$ formaldehyde in the medium at room temperature for 10 min, followed by the addition $125 \mathrm{mM}$ glycine at room temperature for $5 \mathrm{~min}$, and then scraped into sodium dodecyl sulfate (SDS) lysis buffer (50 mM Tris-HCl [pH 8.1], 1\% SDS, and $10 \mathrm{mM}$ EDTA) with antibodies against H3K4me3 and IgG. Soluble lysate was rotated with $2 \mu \mathrm{l}$ antibody overnight at $4{ }^{\circ} \mathrm{C}$ with protease inhibitors $(1 \mu \mathrm{g} / \mathrm{ml}$ leupeptin, aprotinin and pepstatin A, $1 \mathrm{mM}$ phenylmethylsulfonyl fluoride [PMSF]). Immunoprecipitated complexes were eluted with elution buffer (50 mM Tris-Cl [pH 7.5], $1 \mathrm{mM}$ EDTA, 1\% SDS), and cross-links were reversed by incubation for at least $2 \mathrm{~h}$ at $65^{\circ} \mathrm{C}$. DNA was purified by ChIP DNA Clean \& Concentrator Kit (Zymo) and DNA enrichment was measured by quantitative PCR analysis. To analyze the Prdx 6 promoter region, primer sets consisting of the promoter region (position-530 to -328 from the translation start site, forward: 5' - AGCACTGACATTGGTACGGG-3', reverse: 5'AGCTCCCTACAGAGGAGTGG-3') were used. The amplification reaction was performed under the following conditions: 40 cycles of denaturation at $94^{\circ} \mathrm{C}$, annealing at $60^{\circ} \mathrm{C}$, and extension at $72^{\circ} \mathrm{C}$. Disassociation curves were generated after each PCR was run to ensure that a single product of appropriate length was amplified. The mean CT \pm SE was calculated from individual CT values obtained from triplicate determinations per stage. The normalized mean CT was estimated as $\Delta \mathrm{CT}$ by subtracting the mean CT of input from that of the individual region [25].

\section{Statistical analysis}

The experiments were performed in triplicate independent experiments, and data were presented as three repeats from one independent experiment. Data were reported as the mean \pm standard deviation and evaluated by one-way ANOVA. Significant differences were established at $P<0.05$ [25]. Differences in the distribution of the staining score between the groups were assessed using the Mann-Whitney $U$ test. Probability values $(\mathrm{p})$ less than $0.05(P<0.05)$ were considered as statistically significant. Analysis was performed with SPSS software (version 10.0; SPSS, Chicago, IL, USA) [26].

\section{Results}

\section{PRDX6 expression patterns on TMAs}

To identify the potential role of PRDX6 in CRC, we evaluated 40 CRC samples of CRCs stratified by node status tumor tissues. We collected CRC samples from 18 node-negative and 22 node-positive patients (Table 1). Each CRC sample was paired with a duplicate from the same patient at the time of resection. Significance analysis of microarrays was used to evaluate each intergroup. We further investigated the correlation between PRDX6 expression and node status in a larger, independent patient cohort by immunohistochemical staining of TMAs. TMAs from two sources, representing 40 eligible cases from different geographic regions in a limitation, were analyzed. Fig. 1 shows the representative patterns of PRDX6 staining in the cytoplasm. Using a $0-5$ scale representing a combination of staining intensity and fraction of cells stained, an increased frequency of high-level PRDX6 expression in nodepositive CRC was seen in this intergroup. The distribution of scores for the node-negative and node-positive groups was significantly different statistically, based on a Mann-Whitney $U$ test $(P=0.03$; Table 2) [26]. These findings indicate that PRDX6 expression might contribute to CRC progression and be a potential therapeutic target of this disease. 
Tumorigenicity

Effects of PRDX6 on cell invasion and survival

To establish stable colon cancer cell lines continuously to determine the potential mechanisms by which PRDX6 might promote metastasis in CRC, HCT-116 cells were transfected with PRDX6 short hairpin RNA (shRNA) or scrambled shRNA lentiviral particles. We next studied the effect of an altered PRDX6 expression level on biologic processes that are relevant to metastasis in human HCT-116 stably expressing PRDX6. We determined a number of CRC cell lines for the PRDX6 level and selected HCT116 or DLD-1 for studies based on their moderate levels of endogenous PRDX6 expression that could be operated genetically. From our study, using the scratch-wound assay, with a lasting rapid movement, down-regulation of PRDX6 by specific shRNAs (siPRDX6) resulted in a marked reduction of the HCT-116 cell migration front compared with the control siRNAtransfected (siControl) or untreated group (Control) at $24 \mathrm{~h}{ }^{* *} \mathrm{p}<0.05$, Fig. 2A). The Boyden chamber assay was used to evaluate the in vitro migration and invasion effect of siPRDX6 associated with the aggressive status of siControl HCT-116 cells. Knockdown of PRDX6 reduced the invasion by more than $30 \%$ in the siControl-transfected cells and by $25 \%$ in the control cells $\left(^{* *} p<0.05\right.$, Fig. $\left.2 B\right)$. The EMT is the cell intermediate filament system of switches that connects to adherens junctions and to focal adhesions [27]. Typical elements of the EMT include loss of expression of proteins associated with adhesive junctions (such as E-cadherin). Down-regulation of PRDX6 significantly increased the expression of E-cadherin compared with the sicontrol-transfected cells or the untreated group (Control) (Fig. 2C). However, while transient transfection with PRDX6 recovered plasmid in the PRDX6+siPRDX6 group, it significantly restored the capability of CRC invasion, survival and E-cadherin expression to HCT-116 siPRDX6 knockdown cells (Fig. 2).

Effects of PRDX6 expression on cell cycle checkpoint and epithelial mesenchymal markers

The eukaryotic cell cycle control system incorporates a complex process that monitors and dictates the progression by which these checkpoints are frequently dysregulated in cancer [28]. Cell cycle analysis to assess whether PRDX6 is involved in the cell cycle distribution of HCT-116 cells revealed that siPRDX6-transfected cells induce G1/S arrest in HCT-116 cells. These data suggest that PRDX6 inactivation induces G1 and S arrest by $75 \%$ and $12 \%$, respectively. Similarly, as expected, transient transfection with PRDX6 reversed to HCT-116 siPRDX6 knockdown cells by approximately 58\% and 21\% in the PRDX6+siPRDX6 group (Fig. 3A). PRDX6, is highly expressed in cancer cells and transcriptionally regulated by various oxidative stresses and signaling regulators [29]. PRDX6 plays an important role in the oxidative stress response, as an anti-apoptotic function, and then loss of Prdx6 increases susceptibility to oxidative stress [30]. Previous studies found that peroxiredoxin (PRDX6) 


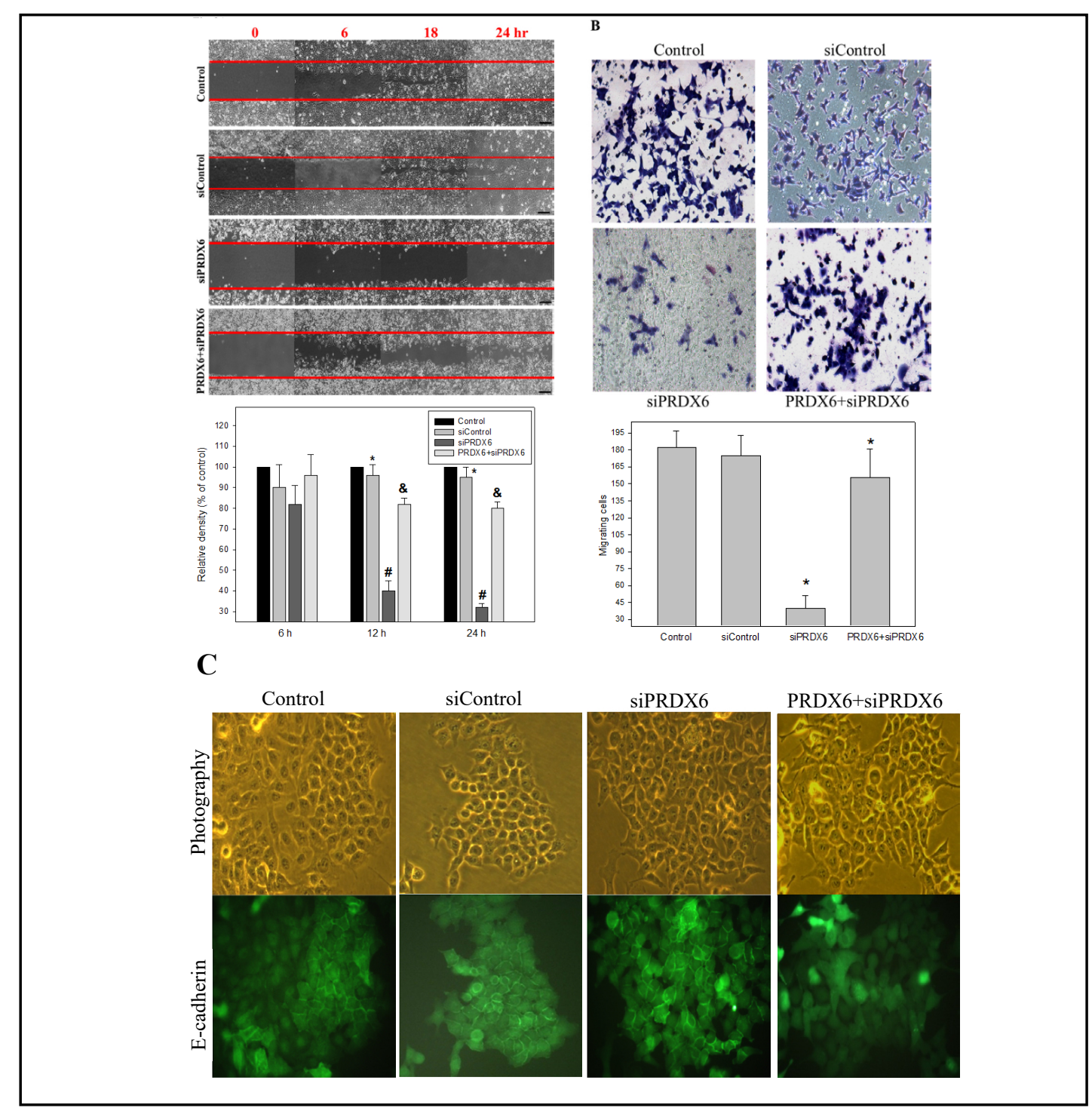

Fig. 2. Effects of PRDX6 inactivation on in vitro cell migration and invasiveness of human colorectal cancer cells. (A) HCT-116 cells were transfected with lentiviral shRNA targeting PRDX6 (siPRDX6) or nontargeting control (siControl); meanwhile, the cells were transfected with PRDX6 plasmid followed by $24 \mathrm{~h}$ (PRDX6+siPRDX6) and the migration for 6, 12 and $24 \mathrm{~h}$ using the scratch-wound assay was visualized as described in "Methods." The percentage of surface area filled by the HCT-116 cells was subsequently quantified by densitometric analyses relative to the control, which was set at $100 \%$ in the photograph. Data are presented as means \pm SD based on three independent experiments. The experiments were performed in triplicate, and data are presented as means $\pm \mathrm{SD}$. ${ }^{\mathrm{P}}<0.05$, compared with the control group for $6 \mathrm{~h}$. \#P<0.05, compared with the control group for 12 h. \&P<0.05, compared with the control group for 24 h. (B) PRDX6 knockdown on invasiveness of HCT-116 cells. Cells were transfected with various shRNA. Invasion for 24 $\mathrm{h}$ through a layer of matrigel was determined by the Boyden Chamber method as described in "Methods." The lower and upper chemotaxis cells were separated by a polycarbonate membrane. Microscopy images detected cells that migrated into the inner membrane. Magnification: $\times 200$. The cell migration was quantified by counting the number of cells that migrated into the inner membrane. The control cells remained untreated. The experiments were performed in triplicate, and data are presented as means \pm SD. The symbol * indicates means that are significantly different when compared to the control group with $\mathrm{P}<0.05$, respectively. (C) HCT-116 cells were transfected with various shRNA for the indicated times, and immunofluorescence analysis showed expression of markers E-cadherin in the parental cell line of HCT-116 as described in "Materials and Methods".

\section{KARGER}


Huang et al.: PRDX6 Expression May Participate in the Regulation of the CRC Tumorigenicity

Fig. 3. Effect of PRDX6 inactivation on cell cycle distribution in HCT116 cells. (A) After treatment with shRNA for $24 \mathrm{~h}$, the cells were fixed and stained with propidium iodide, and the DNA content was analyzed by flow cytometry (FACS). The cell number percentage in each phase (G1, S, and G2/M) of the cell cycle was calculated and (B) intracellular ROS were determined by FACS analysis as described in "Materials and Methods." Representative histograms were typical for H2DCFDA profiles. The production of ROS was expressed as the fold of the control group. (C) Lysates from control, siPRDX6 and PRDX6+siPRDX6 clones of HCT-116 cells were used for western blot analysis. Data presented in the western blot are derived from a representative study for the indicated time 24 h. The association of PRDX6 with Nox1 was determined by immunoprecipitation followed by western blot with antibody.

6 participates in activation of NADPH oxidase (Nox) enzymes in human endothelial cells. Nox1 complex is most abundant in colon epithelial cells $[31,32]$ and participates in mucosal cell migration, wound healing, and epithelial homeostasis, which binds to PRDX6 and activate NADPH oxidase Nox1. We also investigated the association of PRDX6 with the Nox1 complex, whether Prdx6 may have increasing effects on superoxide generation

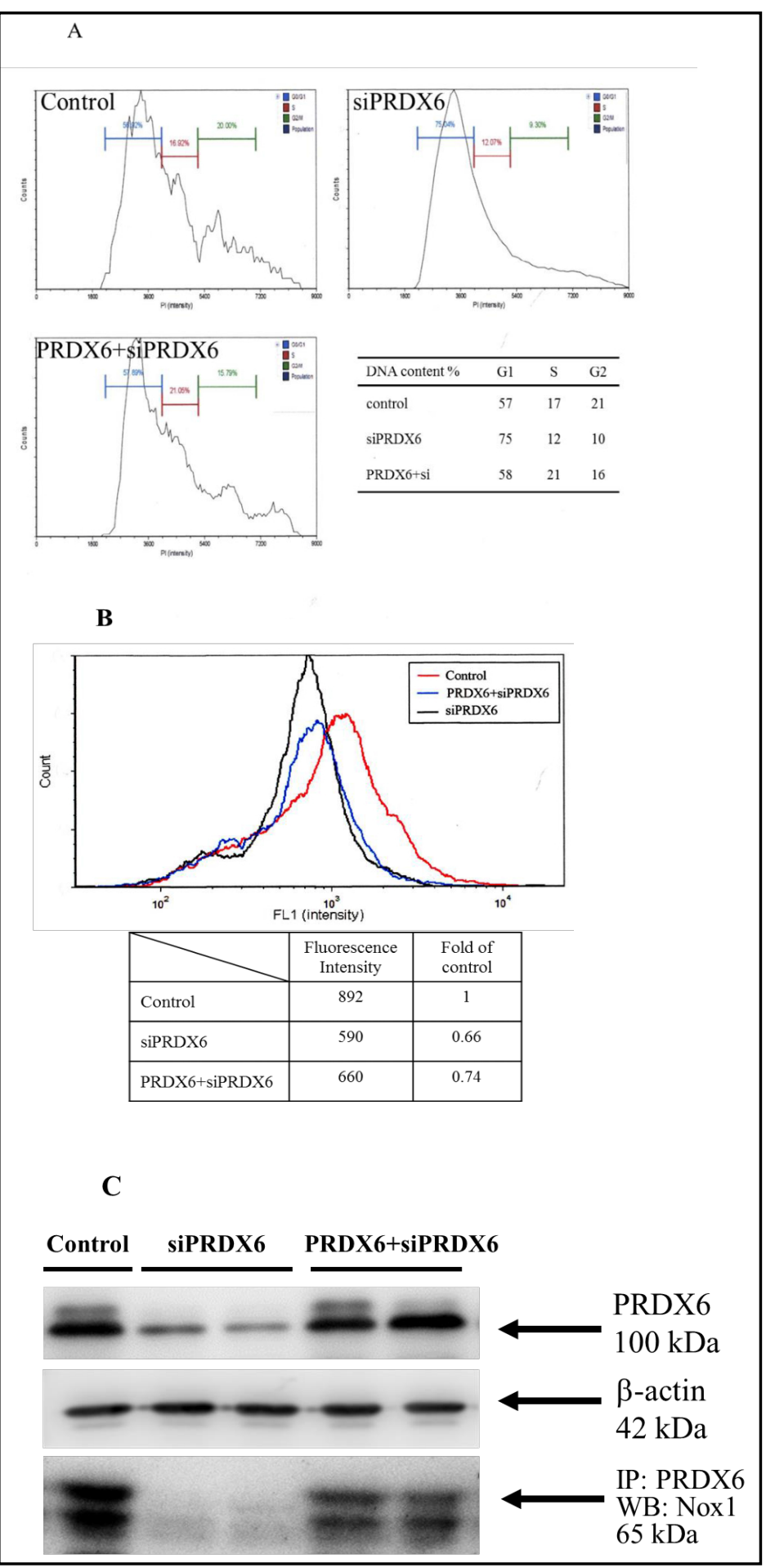

by Nox1. Extracellular

superoxide release was detected by using the fluorescent probes of H2DCFDA. The results of down-regulation of PRDX6 (siPRDX6) reduced the generation of ROS compared to the invasiveness of the untreated group (Control) HCT-116 cells, indicating the involvement of PRDX6 in cell sensitivity by free radicals. Transient transfection with PRDX6 rescue plasmid restored free radical susceptibility of the PRDX6+siPRDX6 group cells (Fig. 3B). In addition, the results of down-regulation of PRDX6 reduced the Prdx6 and Nox1 protein 
Fig. 4. PRDX6 inactivation inhibited tumorigenicity and metastasis in vitro and modulation of cell cycle-related proteins in HCT-116 cells. Lysates from control, siControl, siPRDX6 and PRDX6+siPRDX6 clones of HCT-116 cells were used for Western blot analysis. Data presented in the Western blot are derived from a representative study, and comparisons of protein expression are calculated from three replicate experiments for the indicated time of $18 \mathrm{~h}$. The effect of PRDX6 knockdown on the protein levels of PRDX6,

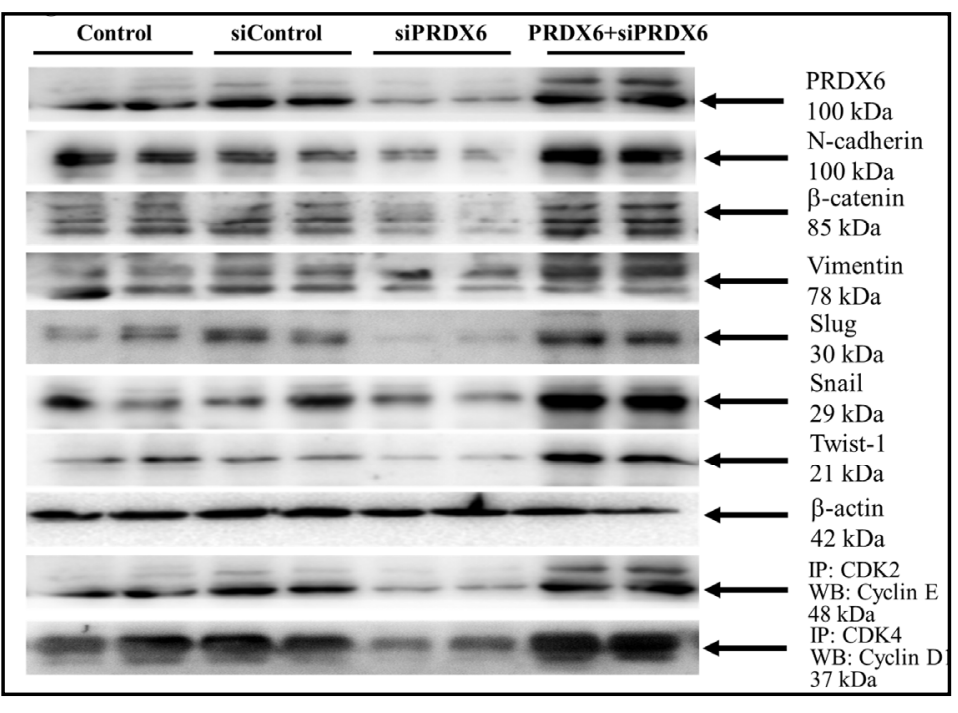
$\mathrm{N}$-cadherin, b-catenin, Vimentin,

Slug, Snail, Twist- 1 and $\beta$-actin were detected with the indicated antibodies. The association of CDK2 with Cyclin E and the association of CDK4 with Cyclin D1 were determined by immunoprecipitation followed by Western blot with antibody.

Fig. 5. Effect of PRDX6 inactivation on the activation of $\mathrm{PI} 3 \mathrm{~K} / \mathrm{AKT} / \mathrm{p} 38$ pathway and expression of p50 NFkB. HCT-116 cells were transfected with lentiviral shRNA PRDX6 (siPRDX6) or nontargeting control (siControl); meanwhile, the cells were transfected with PRDX6 plasmid, PRDX6+siPRDX6. Whole cell lysate proteins for the indicated time of $18 \mathrm{~h}$ were prepared and

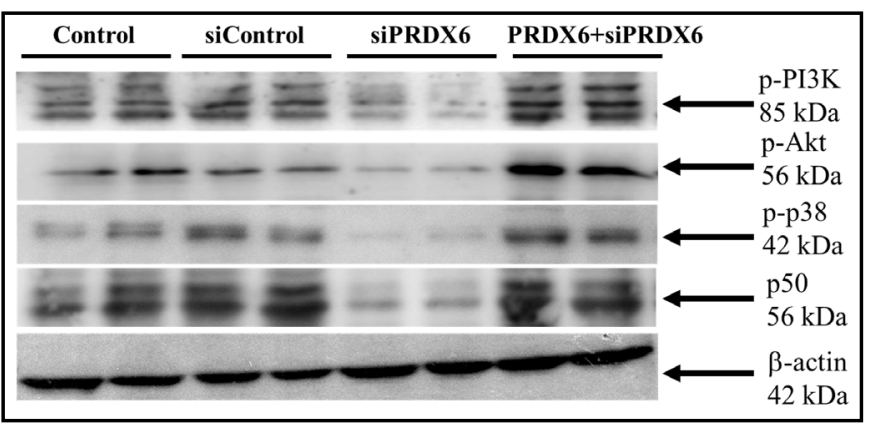
analyzed by Western blot, with $\beta$-actin serving as the loading control. Cell lysates were analyzed by $10 \%$ SDS-PAGE and subsequently treated with antibodies against phosphorylation of PI3K, AKT, p38, p50 and NFKB.

levels in these cells when compared with HCT-116 cells (CL). In this case, transient transfection with PRDX6 rescue plasmid restored PRDX6 and Nox1 components and superoxide generation when compared with siPRDX6 transfectant (Fig. 3C), indicating the activation of PRDX6 led to higher levels of these Nox1 components and higher superoxide generation. However, further findings may determine that PRDX6 promotes cell motility, migration, and invasion and ROS production in HCT-116 cells and then aberrant ROS, such as the superoxide radical contribution to cancer progression and metastases is mostly due to ability to affecting EMT via phosphoinositide 3-kinase, and Akt signaling [33]; therefore, PRDX6 was the most abundant and widely distributed member of the mammalian Prxs [29, 30 ,34]. The pathophysiological role of CRC in its appearance in tumor cells of epithelial origin is consistent with the engagement of the EMT, but the process by which tumor cells acquire a more aggressive phenotype remains unclear. The Western blotting assay was used to evaluate the EMT-related proteins in in vitro migration and the invasion effect of PRDX6 with the aggressive status of HCT-116, siControl, siPRDX6 as well as PRDX6+siPRDX6 cells. Epithelial-mesenchymal transition (EMT) is a key developmental process often activated during cancer invasion and metastasis [35]. As shown in Fig. 4, these results demonstrated that the PRDX6 shRNA can effectively knock down PRDX6 expression at the protein level and be used to study PRDX6-mediated effects. PRDX6 inactivation significantly decreased the 


\section{Cellular Physiology Cell Physiol Biochem 2018;51:2616-2630 \begin{tabular}{l|l|l} 
and Biochemistry & $\begin{array}{l}\text { DOI: 10.1159/000495934 } \\
\text { Publisned onIIne:T1 December } 2018\end{array}$ & $\begin{array}{l}\text { (c) } 2018 \text { The Author(s). Published by S. Karger AG, Basel } \\
\text { www.karger.com/cpb }\end{array}$
\end{tabular} \\ Huang et al.: PRDX6 Expression May Participate in the Regulation of the CRC Tumorigenicity}

Fig. 6. Effect of the kinase inhibitors in blocking histone modification of PRDX6 promoter. HCT-116 cells were incubated with various concentrations of the specific inhibitors NAC, wortmannin and SB203580 for $24 \mathrm{~h}$. Next, the chromatin immunoprecipitation (ChIP) assays were performed by using antibodies against Histone H3 (H3K4me3) to pull down associated DNA. The precipitated DNA was amplified by PCR using primer sets specific to the target sites (-530/-375) of PRDX6 promoter. Input DNA was amplified as a loading control. DNA pulled down by the anti-IgG antibody was served to identify background amplification. The quantitative data were presented as the mean of three repeats from one independent experiment. The data were presented as mean \pm SD of three independent experiments. ${ }^{*} \mathrm{P}<0.05$, compared to the control group.

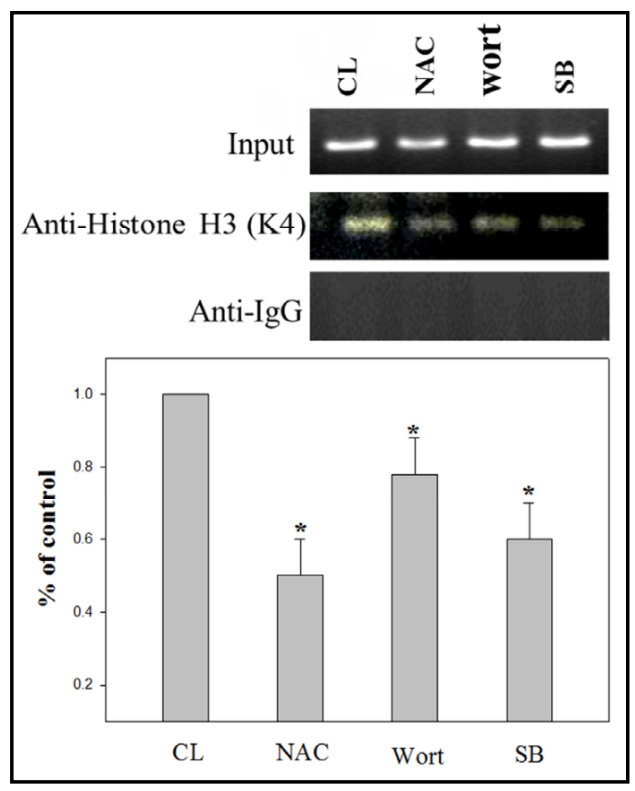

expression of EMT-related proteins, including N-cadherin, b-catenin, Vimentin, Slug, Snail, and Twist-1, rather than in the PRDX6-transfected siPRDX6 cells, PRDX6+siPRDX6 group. The results from these experiments confirmed that PRDX6 expression has a pivotal role in tumor metastasis, showing that it is actively implicated in the phenotype and function of EMT during cell proliferation and metastasis initiation in CRC cancer progression.

PI3K/Akt/p38 signaling pathways mediated histone modification of PRDX6 promoter

Previous studies have shown that EMT activation involves more pleiotropic signals, as in the case of reactive oxygen species produced in response to signal transducers [36]. EMT can be regulated by several signaling pathways including SMADs, signal transducers and activators of phosphoinositide 3-kinase (PI3K)/Akt, and mitogen-activated [37, 38]. Therefore, in this study, we aimed to examine whether the cross talk between the p38 and PI3K/Akt pathways could mediate the EMT process by regulating PRDX6 expression in HCT-116 cells. To evaluate the effect of an altered PRDX6 expression level on biologic processes relevant to the molecular mechanism involved in the PI3K/Akt/p38 signaling pathways of CRC, we checked the protein levels p-PI3K, p-Akt, p-p38 and NFkB p50 in HCT116 cells by Western blot analysis. We observed that PRDX6 shRNA significantly increased the expression of p-PI3K, p-Akt, p-p38, NFkB p50 in human HCT-116 and siControl, which are widely used as a model for studies of cell signaling and invasion (Fig. 5). To elucidate the exogenous PRDX6 transfected cell lines, we transfected with the PRDX6 expression vector in siPRDX6 HCT-116 cells (RDX6+siPRDX6). We found that these cells exhibited markedly increased protein levels p-PI3K, p-Akt, p-p38 and NFkB p50 (Fig. 5). Previous study has shown that methylation modifiers also modulate methylation at histone H3 lysine 4 (H3K4me3), including key effectors and components in cellular PI3K/Akt/p38 signaling pathways that regulate cell proliferation, growth, and survival [39]. To investigate the role of the PI3K/Akt/p38 signaling pathways or endogenous ROS in regulating histone modification of PRDX6 promoter, we performed ChIP to analyze the change in histone methylation (H3K4me3) following treatment with HCT-116 cells [40]. As shown in Fig. 6, HCT-116 cell lines were incubated with inhibitors, including ROS scavenging NAC $(1 \mathrm{mM})$, PI3K/AKT inhibitor wortmannin $(10 \mu \mathrm{M})$, and p38 MAPK inhibitor SB203580 $(30 \mu \mathrm{M})$. This resulted in a decreased trimethylation of histone H3 lysine 4 (H3K4me3), a well-known marker of transcriptional activation. Taken together, the results showed that PRDX6 expression in HCT116 cells is essential to involvement in the PI3K/Akt/p38 signaling pathways or endogenous ROS signaling mediated cell survival and metastasis. 


\section{Cellular Physiology Cell Physiol Biochem 2018;51:2616-2630 \\ and Biochemistry \begin{tabular}{l|l} 
Dublished onlIne: T1 December 2018 & $\begin{array}{l}\text { (c) } 2018 \text { The Author(s). Published by S. Karger AG, Basel } \\
\text { www.karger.com/cpb }\end{array}$ \\
\hline
\end{tabular} \\ Tumorigenicity}

\section{Discussion}

CRC is one of the most commonly occurring malignant tumors in the digestive tract. CRC has posed a substantial threat to the health of numerous patients worldwide. Although important advances have been made in the area of CRC treatment methods, the promising molecular biomarkers involved in the diagnosis, prognosis and prediction in early- or latestage CRC remain rather poor [41,42]. Peroxiredoxins (PRDXs) are a ubiquitously expressed family of small $(22-27 \mathrm{\kappa Da})$ non-seleno peroxidases that catalyze the peroxide reduction, currently known to possess six isozymes, namely, PRDX1-6 in mammalian systems. PRDXs are also a novel sensitive and highly specific marker for metastatic tumors in in vitro or in vivo models [30]. Previous studies have reported findings in regard to the high expressions of PRDX6 in certain cells, indicating that PRDX6 may be involved in the cancer's development [29]. Based on these reports, this study aimed to survey the effects of PRDX6 expression in tissues on the diagnosis and prognosis in human CRC specimens of positive lymph nodes, which distinguish stage I/II from stage III/VI CRC. We used IHC of TMAs as a high throughput method, containing 40 tissue specimens to evaluate the correlation between PRDX6 and node status (Table 1, Fig. 1). Our study's results indicated PRDX6 as showing significant promise as a novel marker for the diagnosis and prognosis of CRC patients (Table 2, Fig. 1). It has been previously reported that epithelial-mesenchymal transition (EMT), which modulates cancer progression and metastasis, has been involved in aberrant ROS levels driving signaling pathways due to a loss of proper redox control [43]. Their contribution to cancer progression and metastases is mainly due to their association with cell proliferation, EMT and apoptosis through PI3K/AKT/p38/p50 [38]. Furthermore, previous studies also demonstrated that abnormal expression of PRDX6, an indication of its tumor-promoting effects, was found in breast cancer, bladder cancer, ovarian cancer, pancreatic cancer and other tumors $[44,45,46,47]$. PRDX6 is an important regulator of the invasiveness state and survival, not only in several types of normal cells, but also in cancer cells, and it possibly indicates a worse prognosis of clinical prostate cancer with radical prostatectomy [46]. In this study, we established a knockdown of the PRDX6 cell line, siPRDX6, and evaluated the biological characters' cell invasion, survival and EMT-associated markers. Interestingly, we determined that inactivated PRDX6 cells significantly showed more features associated with decreasing migratory and invasive potential compared to parental cells in vitro (Fig. 2). The alteration of PRDX6 in siPRDX6 cells was found to be associated with the downregulation of the expression of N-cadherin, $\beta$-catenin, Vimentin, Slug, Snail and Twist- 1 , and inactivation of the PI3K/AKT/p38/p50 pathways (Fig. 4, Fig. 5). PRDX6, a promoter-selective regulator of transcription, is distributed in all organs and essentially in all cell types [40]. Having an ability to regulate the transcription of other genes and the tumor microenvironment, the TGF- $\beta$ /SMAD signaling pathway and the epigenetic pathway provide a mechanism by which PRDX6 might broadly influence the processes relevant to metastasis. Further study will be needed to determine whether PRDX6 is mediated [7].

TGF- $\beta$ /SMAD signaling has a complex role in CRC progression and diagnosis. It has a role in the activation of the p38-MAPK and PI3K/Akt, and changes in the expression of these cell-cycle regulatory proteins might decrease the phosphorylation of Rb that results in G1-S transition and cell proliferation $[14,28]$. Studies in a variety of experimental systems have demonstrated ROS induction and activation of TGF- $\beta /$ PI3K/AKT/p38 and their involvement in the modulation of gene transcription [7]. ROS levels and thereby abnormal expression and activation of NFKB p50 may be a major underlying mechanism for pathologic and abnormal physiologic changes in cells with induced levels of cytoprotective protein PRDX6. Additionally, the PI3K/AKT/p38/p50NFkB pathway might be directly induced to leading to CRC cell cycle proliferation and invasion $[38,39]$. Previous findings suggested that the deregulation of cyclin D1 and cyclin E complexes' expression and activation directly lead to some cancer hallmarks by inducing cell cycle distribution $[14,28]$. More-recent studies that we have conducted have also defined PRDX6 inactivation induced cell cycle G1 arrest with a decrease in the association of the CDK2/cyclin E and CDK4/cyclin D1 complexes directly 
Huang et al.: PRDX6 Expression May Participate in the Regulation of the CRC

Tumorigenicity

implicated in cell survival, which is consistent with previous reports. Consistently, our present work demonstrated that PRDX6 re-expression accelerated the cell cycle transition, which is similar to the effects shown in numerous other studies with respect to PRDX6 control of tumorigenesis (Fig. 3). Numerous histone post-translational modifications contribute to chromatin and gene regulation, the tri-methylation of histone 3 lysine 4 (H3K4me3) is a wellestablished marker of active transcription [48] and associated enzymes in the pathogenesis of CRC have been implicated in oncogenesis and cancer-related functions [49]. Histone methylation not only regulates many biological functions, including gene transcription, nucleosomal positioning, DNA replication and repair, but also influences the carcinogenesis of cancers by affecting various cancer pathways. Moreover, we have shown the role of PRDX6 in mediating CRC development via ROS and PI3K/Akt signaling pathways involved in methylation at histone $\mathrm{H} 3$ lysine 4 (H3K4) of PRDX6 promoter transcriptional activation in HCT-116 cells (Fig. 6) [40,44]. Several studies have reported increases in ROS levels leading to a number of human diseases, such as cancer, because of over-ROS-mediated PRDX6 expression and activation of such factors as TGF- $\beta$ and PI3K/AKT/p38/p50 pathways; it has also been shown that PRDX6 promoter has various putative regulatory elements $[7,13,37]$. Further work is needed to explore the role of transcriptional factors, particularly in PRDX6 regulation. A cohort study with a greater number of human CRC tissues and an examination to investigate the association with invasiveness are also needed.

Recently, peroxiredoxins have an important function in carcinogenesis, suggesting a possible role in cancer cell maintenance. Park et al. [29] have demonstrated that the dual action of PRDX6 in tumorigenesis, depending on increased level of PRDX6. We determined that an attempt to identify PRDX6 and validated that the phenotype in CRC is offered by the modulation of PRDX6. We hypothesized that CRC cells would exhibit up-expression of PRDX6 in otherwise normal cells may be tumorigenic in CRC tissues compared with metastasis of colorectal adenomas in node-positive CRC, as observed in immunohistochemical staining of tissue microarrays while loss of PRDX6 suppressed PI3K/AKT/p38/p50 signaling pathways that resulted in down-regulation of epithelial-mesenchymal transition (EMT) proteins N-cadherin, $\beta$-catenin, Vimentin, Slug, Snail and Twist-1. PRDX6 suppression in CRC cells alter cell-cycle regulatory proteins CDK2/cyclin E and CDK4/cyclin D1 complex leads to inhibition of tumorigenicity, migration, invasion, and metastasis. In this study, we determined by immunohistochemical staining of CRC TMAs that in the case of HCT-116 cells, a loss of PRDX6 expression in CRC promotes cancer cell progression and metastasis. The present investigation provides a molecular mechanism demonstrating the role of PRDX6 in mediating CRC cancer cell proliferation and migration/invasion. PRDX6 overexpression in many human cancers, serving a potential prognostic marker, regulated cell-cycle regulatory proteins and its transcriptional induction by oxidative stress via increased activity of redoxsensitive transcription factors and activation of PI3K/AKT/p38/p50 signaling pathways, which are able to interact with the PRDX6 gene promoter region and/or increased oncogenic activity present in cancer cells leading to cancer progression [29, 34, 44, 34]. These data suggest that the levels of PRDX6 and Nox1 components are closely linked positively regulates NADPH oxidase Noxa1 activity involved in superoxide generation (Fig. 3B, Fig. 3C). PRDX6 expression serve as a biomarker of node-positive status and may have a role as an important endogenous regulator of cancer cell tumorigenicity in CRC through the activation of endogenous ROS and the PI3K/AKT/p38/p50 pathways. Recently, the NADPH oxidase (Nox1 enzyme complex) was shown to direct colon epithelial cell migration and wound healing through the VEGF receptor type2 (VEGR2, KDR/Flk1) and Akt, p38 MAP kinase and PAK (p21-activated kinase) mediated pathways previously, plays a critical role in the correlation with CRC cancer migration, invasion and epithelial-mesenchymal transition [48]. Nox1 is most abundant in colon epithelial cells and participates in epithelial cell migration, while inappropriate or excessive ROS production can damage surrounding tissues and promote inflammation, the activity of NADPH oxidases is subject to tight regulation [50]. Here, we show PRDX6 as a novel regulator of Nox1-based superoxide generation and activated cells survival and aggressive pathways in colorectal cancer cells, which appears to involve the ROS 


\section{Cellular Physiology and Biochemistry \\ Tumorigenicity}

and PI3K/Akt/NFkB signalling pathways and that PRDX6 was involved in methylation at histone H3 lysine 4 (H3K4) of PRDX6 promoter in HCT-116 cells. In addition, Prdx 6 deficiency results in destabilization of Nox1 components, whereas PRDX6 rescue plasmid restored increasing levels of oxidase activity. These results highlight a novel pathway in which PRDX6 supports the oxidant-generating activity of Nox1 to promote cell migration which PRDX6 recruitment into the Nox1 complex. PRDX6 modulates Nox1dependent activation and migration of HCT-116 cells and then future work determine whether proposes that PRDX6 has a role in the expression of NOX1 protein, which may suggest novel strategies for therapeutic intervention.

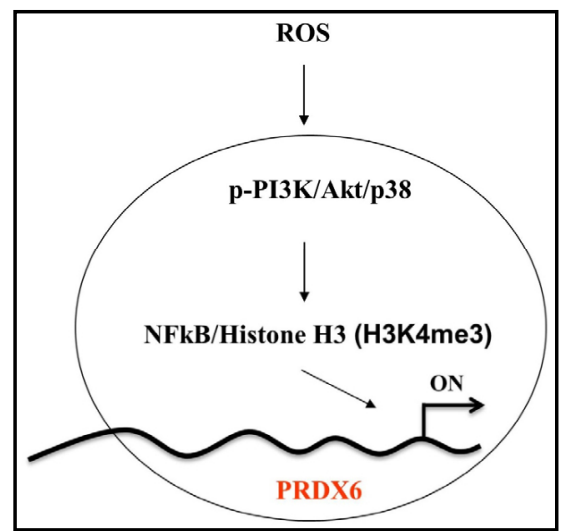

Fig. 7. Schematic presentation of the histone modification (H3K4me3) of PRDX6 promoter by endogenous ROS and PI3K/Akt/NFkB signaling pathways in HCT-116 cells.

\section{Conclusion}

Our results imply that PRDX6 mediated cell survival and aggressive pathways in colorectal cancer cells via ROS and PI3K/Akt/NFkB signaling pathways and that PRDX6 was involved in methylation at histone $\mathrm{H} 3$ lysine 4 (H3K4) of PRDX6 promoter in HCT-116 cells (Fig. 7). This study provides evidence that PRDX6 expression in established CRC cell lines influenced invasion, survival, and EMT, and it also shows that PRDX6 may participate in the regulation of the tumorigenicity.

\section{Acknowledgements}

Funding for this study was provided in part by research grants BMRPD42, CLRPG8G0591, CMRPG6E0191， CMRPG6E0192， CMRPG6H0031， CMRPG6H0032， CMRPF6G0011, CMRPF6G0012, CMRPF6G0013 from Chang Gung Memorial Hospital, Chiayi, Taiwan, and Chang Gung University of Science and Technology, Chia-Yi Campus, Taiwan, and by the Ministry of Science and Technology, Taiwan (MOST 107-2320-B-255 -001 -MY3).

\section{Disclosure Statement}

There is no financial/commercial conflict of interests.

\section{References}

$\rightarrow 1$ Brenner H, Kloor M, Pox CP: Colorectal cancer. Lancet 2014;383:1490-1502.

-2 Jonker DJ, O'Callaghan CJ, Karapetis CS, Zalcberg JR, Tu D, Au HJ, Berry SR, Krahn M, Price T, Simes RJ, Tebbutt NC, van Hazel G, Wierzbicki R, Langer C, Moore MJ: Cetuximab for the treatment of colorectal cancer. N Engl J Med 2007;357:2040-2048.

3 Siegel R, Naishadham D, Jemal A: Cancer statistics, 2012. CA Cancer J Clin. 2012;62:10-29.

4 Haggar FA, Boushey RP: Colorectal cancer epidemiology: incidence, mortality, survival, and risk factors. Clin Colon Rectal Surg 2009;22:191-197.

5 Compton CC: Optimal pathologic staging: defining stage II disease. Clin Cancer Res. 2007;13:6862s-6870s.

6 Chiang AC, Massagué J: Molecular basis of metastasis. N Engl J Med 2008;359:2814-2823.

7 Tse JC, Kalluri R: Mechanisms of metastasis: epithelial-to-mesenchymal transition and contribution of tumor microenvironment. J Cell Biochem 2007;101:816-829. 


\section{Cellular Physiology Cell Physiol Biochem 2018;51:2616-2630 \begin{tabular}{l|l|l} 
DOI: 10.1159/000495934 & (c) 2018 The Author(s). Published by S. Karger AG, Basel
\end{tabular} and Biochemistry Published on Ine: IT vecember 2018 www.karger.com/cpb}

Huang et al:: PRDX6 Expression May Participate in the Regulation of the CRC

Tumorigenicity

8 Sillars-Hardebol AH, Carvalho B, de Wit M, Postma C, Delis-van Diemen PM, Mongera S, Ylstra B, van de Wiel MA, Meijer GA, Fijneman RJ: Identification of key genes for carcinogenic pathways associated with colorectal adenoma-to-carcinoma progression. Tumour Biol 2010;31:89-96.

-9 Hall A, Nelson K, Poole LB, Karplus PA: Structure-based insights into the catalytic power and conformational dexterity of peroxiredoxins. Antioxid Redox Signal 2011;15:795-815.

10 Lu W, Fu Z, Wang H, Feng J, Wei J, Guo J: Peroxiredoxin 2 is upregulated in colorectal cancer and contributes to colorectal cancer cells' survival by protecting cells from oxidative stress. Mol Cell Biochem 2014;387:261-270.

11 Dammeyer P, Arnér ES: Human Protein Atlas of redox systems - what can be learnt? Biochim Biophys Acta 2011;1810:111-138.

-12 Raatikainen S, Aaaltomaa S, Kärjä V, Soini Y: Increased Peroxiredoxin 6 Expression Predicts Biochemical Recurrence in Prostate Cancer Patients After Radical Prostatectomy. Anticancer Res 2015;35:6465-6470.

13 Xu J, Lamouille S, Derynck R: TGF-beta-induced epithelial to mesenchymal transition. Cell Res 2009;19:156-172.

14 Duronio RJ, Xiong Y: Signaling pathways that control cell proliferation. Cold Spring Harb Perspect Biol 2013;5:a008904.

15 Garcia BA, Hake SB, Diaz RL, Kauer M, Morris SA, Recht J, Shabanowitz J, Mishra N, Strahl BD, Allis CD, Hunt DF: Organismal differences in post-translational modifications in histones $\mathrm{H} 3$ and H4. J Biol Chem 2007;282:7641-7655.

16 Lin Y, Buckhaults PJ, Lee JR, Xiong H, Farrell C, Podolsky RH, Schade RR, Dynan WS: Association of the actin-binding protein transgelin with lymph node metastasis in human colorectal cancer. Neoplasia 2009;11:864-873.

17 Wang Z, Jiang L, Huang C, Li Z, Chen L, Gou L, Chen P, Tong A, Tang M, Gao F, Shen J, Zhang Y, Bai J, Zhou M, Miao D, Chen Q: Comparative proteomics approach to screening of potential diagnostic and therapeutic targets for oral squamous cell carcinoma. Mol Cell Proteomics 2008;7:1639-1650.

18 Lee KC, Kuo HC, Shen CH, Lu CC, Huang WS, Hsieh MC, Huang CY, Kuo YH, Hsieh YY, Teng CC, Lee LY, Tung SY: A proteomics approach to identifying novel protein targets involved in erinacine A-mediated inhibition of colorectal cancer cells' aggressiveness. J Cell Mol Med 2017;21:588-599.

19 Kuo HC, Kuo YR, Lee KF, Hsieh MC, Huang CY, Hsieh YY, Lee KC, Kuo HL, Lee LY, Chen WP, Chen CC, Tung SY: A Comparative Proteomic Analysis of Erinacine A's Inhibition of Gastric Cancer Cell Viability and Invasiveness. Cell Physiol Biochem 2017;43:195-208.

-20 Tao RR, Wang H, Hong LJ, Huang JY, Lu YM, Liao MH, Ye WF, Lu NN, Zhu DY, Huang Q Fukunaga K, Lou YJ, Shoji I, Wilcox CS, Lai EY, Han F: Nitrosative stress induces peroxiredoxin 1 ubiquitination during ischemic insult via E6AP activation in endothelial cells both in vitro and in vivo. Antioxid Redox Signal 2014;21:1-16.

21 Gao W, Xua J, Wang F, Zhang L, Peng R, Zhu Y, Tang Q, Wu J: Mitochondrial Proteomics Approach Reveals Voltage-Dependent Anion Channel 1 (VDAC1) as a Potential Biomarker of Gastric Cancer. Cell Physiol Biochem 2015;37:2339-2354.

22 Lu CC, Huang WS, Lee KF, Lee KC, Hsieh MC, Huang CY, Lee LY, Lee BO, Teng CC, Shen CH, Tung SY, Kuo HC: Inhibitory effect of Erinacines A on the growth of DLD-1 colorectal cancer cells is induced by generation of reactive oxygen species and activation of p70S6K and p21. J Funct Foods 2016;21:474-484.

-23 Huang WS, Kuo YH, Kuo HC, Hsieh MC, Huang CY, Lee KC, Lee KF, Shen CH, Tung SY, Teng CC: CIL-102induced cell cycle arrest and apoptosis in colorectal cancer cells via upregulation of p21 and GADD45. PLoS One 2017;12:e0168989.

24 Huang WS, Hsieh MC, Huang CY, Kuo YH, Tung SY, Shen CH, Hsieh YY, Teng CC, Lee KF, Chen TC, Lee KC, Kuo HC: The Association of CXC Receptor 4 mediated signaling pathway with oxaliplatin-resistant human colorectal cancer cells. PLOS One 2016;11:e0159927.

25 Huang WS, Chin CC, Chen CN, Kuo YH, Chen TC, Yu HR, Tung SY, Shen CH, Hsieh YY, Guo SE, Shi CS, Liu TJ, Kuo HC: Stromal cell-derived factor-1/CXC receptor 4 and $\beta 1$ integrin interaction regulates urokinase-type plasminogen activator expression in human colorectal cancer cells. J Cell Physiol 2012; 227:1114-1122.

26 Lu CC, Kuo HC, Wang FS, Jou MH, Lee KC, Chuang JH: Upregulation of TLRs and IL-6 as a marker in human colorectal cancer. Int J Mol Sci 2014;16:159-177.

27 Chaffer CL, Weinberg RA: A perspective on cancer cell metastasis. Science. 2011;331:1559-1564.

28 Malumbres M, Barbacid M: Cell cycle, CDKs and cancer: a changing paradigm. Nat Rev Cancer 2009;9:153166. 


\section{Cellular Physiology Cell Physiol Biochem 2018;51:2616-2630 \begin{tabular}{l|l|l|l} 
DOl: 10.1159/000495934 2018 The Author(s). Published by S. Karger AG, Basel & (c)
\end{tabular} and Biochemistry

Tumorigenicity

29 Park MH, Jo M, Kim YR, Lee CK, Hong JT: Roles of peroxiredoxins in cancer, neurodegenerative diseases and inflammatory diseases. Pharmacol Ther 2016;163:1-23.

30 Neumann CA, Fang Q: Are peroxiredoxins tumor suppressors? Curr Opin Pharmacol 2007;7:375-380.

-31 Kwon J, Wang A, Burke DJ, Boudreau HE, Lekstrom KJ, Korzeniowska A, Sugamata R, Kim YS, Yi L, Ersoy I, Jaeger S, Palaniappan K, Ambruso DR, Jackson SH, Leto TL: Peroxiredoxin 6 (Prdx6) supports NADPH oxidase1 (Nox1)-based superoxide generation and cell migration. Free Radic Biol Med 2016;96:99-115.

-32 Suh YA, Arnold RS, Lassegue B, Shi J, Xu X, Sorescu D, Chung AB, Griendling KK, Lambeth JD: Cell transformation by the superoxide-generating oxidase Mox1. Nature 1999;401:79-82.

-33 Clerkin JS, Naughton R, Quiney C, Cotter TG: Mechanisms of ROS modulated cell survival during carcinogenesis. Cancer Lett 2008;266:30-36.

34 Seo MS, Kang SW, Kim K, Baines IC, Lee TH, Rhee SG: Identification of a new type of mammalian peroxiredoxin that forms an intramolecular disulfide as a reaction intermediate. J Biol Chem 2000;275:20346-20354.

-35 Thiery JP: Epithelial-mesenchymal transitions in development and pathologies. Curr Opin Cell Biol 2003;15:740-746.

-36 Chaffer CL, Weinberg RA: A perspective on cancer cell metastasis. Science. 2011;331:1559-1564.

-37 Stec R, Bodnar L, Smoter M, Mączewski M, Szczylik C: Metastatic colorectal cancer in the elderly: An overview of the systemic treatment modalities (Review). Oncol Lett 2011;2:3-11.

-38 Kucuksayan H, Akca H: The crosstalk between p38 and Akt signaling pathways orchestrates EMT by regulating SATB2 expression in NSCLC cells. Tumour Biol 2017;39:1010428317706212.

-39 Testa JR, Tsichlis PN: AKT signaling in normal and malignant cells. Oncogene 2005;24:7391-7393.

-40 Valekunja UK, Edgar RS, Oklejewicz M, van der Horst GT, O’Neill JS, Tamanini F, Turner DJ, Reddy AB: Histone methyltransferase MLL3 contributes to genome-scale circadian transcription. Proc Natl Acad Sci U S A 2013;110:1554-1559.

-41 Jemal A, Siegel R, Ward E, Hao Y, Xu J, Murray T, Thun MJ: Cancer statistics, 2008. CA Cancer J Clin 2008;58:71-96.

42 Christofori G: New signals from the invasive front. Nature 2006;441:444-450.

-43 Fiaschi T, Chiarugi P: Oxidative stress, tumor microenvironment, and metabolic reprogramming: a diabolic liaison. Int J Cell Biol 2012;2012:762825.

44 Alam H, Gu B, Lee MG: Histone methylation modifiers in cellular signaling pathways. Cell Mol Life Sci 2015;72:4577-4592.

45 Zhang J, Wang K, Zhang J, Liu SS, Dai L, Zhang JY: Using proteomic approach to identify tumor-associated proteins as biomarkers in human esophageal squamous cell carcinoma. J Proteome Res 2011;10:28632872.

-46 Raatikainen S, Aaaltomaa S, Kärjä V, Soini Y: Increased Peroxiredoxin 6 Expression Predicts Biochemical Recurrence in Prostate Cancer Patients After Radical Prostatectomy. Anticancer Res 2015;35:6465-6470.

\$7 Thongwatchara P, Promwikorn W, Srisomsap C, Chokchaichamnankit D, Boonyaphiphat P, Thongsuksai P: Differential protein expression in primary breast cancer and matched axillary node metastasis. Oncol Rep 2011;26:185-191.

-48 Huang T, Lin C, Zhong LL, Zhao L, Zhang G, Lu A, Wu J, Bian Z: Targeting histone methylation for colorectal cancer. Therap Adv Gastroenterol 2017;10:114-131.

49 Bannister AJ, Kouzarides T: Regulation of chromatin by histone modifications. Cell Res 2011;21:381-395.

50 Sadok A, Bourgarel-Rey V, Gattacceca F, Penel C, Lehmann M, Kovacic H: Nox1-dependent superoxide production controls colon adenocarcinoma cell migration. Biochim Biophys Acta 2008;1783:23-33. 